\title{
Numerical investigation of mode transition and hysteresis in a cavity-based dual-mode scramjet combustor
}

\author{
Xu Zhang ${ }^{\mathrm{a}, \mathrm{b}}$, Lianjie Yue ${ }^{\mathrm{a}, \mathrm{b}, *}$, Tinglong Huang ${ }^{\mathrm{a}, \mathrm{b}}$, Qifan Zhang ${ }^{\mathrm{a}, \mathrm{b}}$, Xinyu Zhang ${ }^{\mathrm{a}, \mathrm{b}}$ \\ a State Key Laboratory of High Temperature Gas Dynamics, Institute of Mechanics, Chinese Academy of Sciences, Beijing 100190, China \\ b School of Engineering Science, University of Chinese Academy of Sciences, Beijing 100049, China
}

\section{A R T I C L E I N F O}

\section{Article history:}

Received 10 May 2019

Accepted 18 September 2019

Available online 24 September 2019

\section{Keywords:}

Hysteresis

Dual-mode scramjet

Supersonic combustion

Mode transition

Flame stabilization

Combustion oscillation

\begin{abstract}
A B S T R A C T
The effect of ethylene fuel equivalence ratio (ER) variation directions on combustion states in a dual-mode scramjet combustor was numerically investigated. The combustor employed transverse wall fuel injectors and downstream cavity flameholders without pilot fuel, which are fundamental components in many practical combustors. The isolator inflow Mach number was 3.1, and static pressure, stagnation pressure and stagnation temperature were $53 \mathrm{kPa}, 2622 \mathrm{kPa}$ and $1656 \mathrm{~K}$, respectively. The ER was regulated abruptly in a piecewise constant manner, from 0.10 to 1.02 , and then back to 0.10 . A 3-D URANS method with a recognized two-step kinetics model was adopted. Results exhibited two combustion hysteresis loops, which indicated that different types of combustion mode transitions could result in hysteresis. The first was a hysteretic phenomenon between separated and shock-free scramjet modes based on steady quasi-one-dimensional combustor flow assumptions, and the second was between two different patterns of separated scramjet modes. Hysteresis mechanisms are elucidated from the viewpoint of combustion flow structures. The first hysteresis was attributed to flame stabilization mode transitions between the cavity shear-layer stabilized mode and the jet-wake stabilized mode, along with the transition hysteresis of a pre-combustion shock train's establishment and vanishment. The flame stabilization locations were greatly influenced by the flow separation states ahead of the fuel injectors, and the flow separations were in return determined by the flame distributions. The second hysteresis was attributed to transitions between weak-oscillation mode and intensive-oscillation mode with the transition hysteresis of shock reflection amount increase and decrease of the pre-combustion shock train structure, which were both in the jet-wake stabilized location. Flame in the low-speed region beside the separation bubbles ahead of the fuel injectors provided heat and hot radicals for downstream flame stabilization, and the pre-injector flame intensity greatly influenced the combustion oscillation states.
\end{abstract}

(c) 2019 Elsevier Masson SAS. All rights reserved.

\section{Introduction}

Dual-mode scramjets provide efficient hypersonic airbreathing propulsion. A dual-mode combustor commonly contains a constant-area isolator and a divergent duct. Admittedly, it operates in ramjet (subsonic combustion) mode or scramjet (supersonic combustion) mode [1], based on whether the flow is thermally choked. Mode transition [2-5] is a critical phenomenon that may generate a nonlinear change (abrupt change, catastrophe) of thrust. Meanwhile, researchers found that under a fixed inflow condition, combustion states didn't only depend on the fuel ER, but also on historical ER variation directions (increase or decrease) [5-10].

\footnotetext{
* Corresponding author at: No. 15 Beisihuanxi Road, Beijing, 100190, China. E-mail address: yuelj@imech.ac.cn (L.J. Yue).
}

Dual-mode combustions include complex physical-chemical processes, such as turbulence/chemistry, shock/boundary-layer interactions, etc. These processes are inherently nonlinear. Thus, an ER variation may cause a combustion-state catastrophe. Mathematically, catastrophe is the main reason of hysteresis [11]. In dualmode combustors, if the catastrophe positions where combustion modes transit from each other are different, then a hysteresis loop will be observed [12].

Traditional mode definition as ramjet/scramjet mode was identified by one-dimensional theoretical estimates of thermal choking [1]. Recently, researchers proposed different mode definitions describing combustion states from different viewpoints. Micka and Driscoll found two flame stabilization locations, namely the cavity stabilized mode and the jet-wake stabilized mode [13]. The cavity stabilized mode was subdivided by Wang et al. into the cavity shear-layer stabilized and the combined cavity shear-layer/recircu- 


\section{Nomenclature}

ER equivalence ratio

HRR heat release rate

$M a_{i e}$ the mass-flux averaged Mach number at the isolator exit
RANS Reynolds-averaged Navier-Stokes

THRR total heat release rate in the combustor URANS unsteady RANS

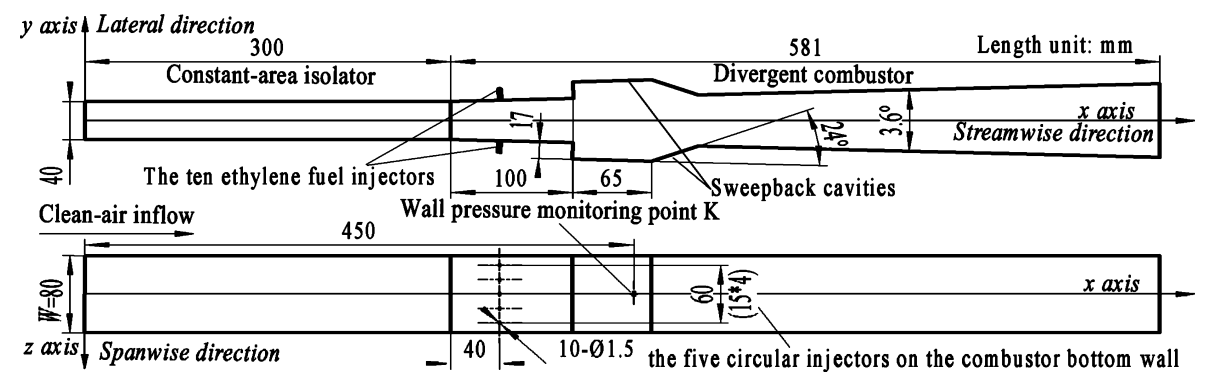

Fig. 1. Schematic diagram of the combustor.

lation stabilized [14]. Mitani et al. defined the lower thrust (weakcombustion) mode and the higher thrust (intensive-combustion) mode [15]. The intensive-combustion mode occurred with large boundary-layer separations ahead of the fuel injectors [16], and Nakaya et al. [17] thought it was equivalent to the jet-wake stabilized mode. The three mode definitions above are steady-state descriptions, but actual combustions are commonly unsteady. Different combustion oscillation patterns have been experimentally confirmed, such as flame oscillations between the jet-wake stabilized and the cavity stabilized locations [13] and thermo-acoustic oscillations in ramjet modes [18].

Under a supersonic inflow condition, flameholders are essential to prevent the flame from being blown out. Flameholder types include ramps, gas-portfires and struts, etc. Rockwell et al. first observed combustion hysteresis in a ramp-based combustor by clean-air tests [5]. They argued the hysteresis occurred in the ramjet/scramjet mode transition region. Wei et al. experimentally studied a gas-portfire-based combustor [6], likewise showed the importance of historical ER paths in the transition region. Bao et al. experimentally studied a strut-based combustor [7], and attributed the hysteresis to transitions between the ramjet modes with one thermal choking point and with two. An obvious hysteresis loop in a strut-based combustor was captured by Zhu et al. under flight Mach 5.0 enthalpy condition, while a much smaller loop under Mach 6.5 [9]. Zhang et al. illustrated a hysteresis behavior in the strut-based combustor by solving the URANS equations [10], and explained it as the transition hysteresis of the pre-combustion shock train.

Cavity flameholders $[19,20]$ are desirable because of low stagnation pressure losses and cooling requirements. Wall normal fuel injections upstream cavities have been adopted in many practical engines. Cavity's recirculation-zone can prolong fuel residence times, and provide hot radicals to stabilize flames in supersonic main flows. Combustion hysteresis phenomena are related to flameholder types, but cavity-based hysteresis had not been surveyed. Dual-mode combustions are intrinsically threedimensional [21] due to corner flows and multi-hole fuel injections, etc. One-dimensional analysis approaches [1,22] commonly assume steady quasi-one-dimensional perfect-gas combustor flows. One-dimensional methods can quickly analyze combustion flows, but large errors are inevitable. Previous studies [5-8] attributed combustion hysteresis to ramjet/scramjet mode transitions by onedimensional analyses with experimental wall-pressure data, but in-depth data depicting shock, boundary-layer and flame were ab- sent. Likewise, in-depth descriptions of cavity-based combustion hysteresis had also not been done yet.

The current numerical study focuses on combustion hysteresis in a cavity-based combustor during a reciprocating fuel ER variation process under flight Mach 6.0 enthalpy condition. Section 2 introduces the combustor configuration, numerical details, and grid convergence and time-step independences validations. Section 3.1 illustrates two combustion hysteresis loops. From the viewpoint of combustion flow structures, hysteresis mechanisms associated with flame stabilization modes and combustion oscillations are elucidated in Sections 3.2 and 3.3, respectively.

\section{Configuration and numerical details}

\subsection{Combustor configuration and inflow conditions}

Fig. 1 illustrates the combustor, which had rectangular crosssections, and was symmetric in both the lateral and spanwise directions. It contained a $300 \mathrm{~mm}$ long constant-area isolator followed by a $581 \mathrm{~mm}$ long $3.6^{\circ}$ divergent duct. The inlet height was $40 \mathrm{~mm}$, and the constant width was $80 \mathrm{~mm}$. Two cavities and ten wall-normal injectors were configured. Each cavity, located $100 \mathrm{~mm}$ downstream the isolator exit, was $66^{\circ}$ aft, $65 \mathrm{~mm}$ long and $17 \mathrm{~mm}$ deep, and spanned the width. The injectors, located $40 \mathrm{~mm}$ downstream the isolator exit, were distributed with an equal spanwise-distance of $15 \mathrm{~mm}$. Each injector had circular cross-sections with the same diameter of $1.5 \mathrm{~mm}$. A point " $\mathrm{K}$ " for wall-pressure monitoring was at the cavity bottom.

The isolator inflow simulated flight Mach 6.0 enthalpy. The inflow contained oxygen and nitrogen, and oxygen mole fraction was $21 \%$. The inflow Mach number was 3.1, and static pressure, stagnation pressure and stagnation temperature were $53 \mathrm{kPa}, 2622 \mathrm{kPa}$ and $1656 \mathrm{~K}$, respectively. The ethylene fuel ER was regulated from 0.10 to 1.02 , and then back to 0.10 . All injectors operated under the same stagnation pressures. The injection stagnation temperature was $300 \mathrm{~K}$ constantly.

\subsection{Numerical method}

Large eddy simulation (LES) and detached eddy simulation (DES) explicitly compute large eddies, giving them advantages in capturing flow instabilities [10]. They received increasing attentions recently [23-25], but excessive computational costs limited their applications. An URANS method cannot predict the full spectrum of turbulent scales, but can predict well the larger scales 
associated with the lower frequency discrete acoustic tones [26]. Han et al. [27] proved an URANS computation could capture the same unsteady flow frequency of $320 \mathrm{~Hz}$ as DES. J. Messineo et al. [28] also certified An URANS tool revealed a combustion oscillation frequency of $470 \mathrm{~Hz}$ which matched the experimental data well. Recently, URANS methods had successfully demonstrated combustion hysteresis or mode transitions [2,10,16,29-32], including main dual-mode combustion features. Low-frequency $(\leq 1000 \mathrm{~Hz})$ largeamplitude oscillations in scramjet combustors are concerned because they can remarkably affect the thrust, and are more likely to cause structure resonances. Meanwhile, 3-D URANS computations cost much time but acceptable [29]. Thus, a 3-D URANS method was adopted in this paper.

For ethylene-air combustions, Westbrook et al. [33] proposed a two-step kinetics model $\left(\mathrm{C}_{2} \mathrm{H}_{4}+2 \mathrm{O}_{2} \rightrightarrows 2 \mathrm{CO}+2 \mathrm{H}_{2} \mathrm{O}, \mathrm{CO}+0.5 \mathrm{O}_{2} \rightleftharpoons\right.$ $\mathrm{CO}_{2}$ ). They experimentally demonstrated this model correctly reproduces flame speeds over wide ranges of ER and combustion pressure. By a 3-D RANS numerical method with this kinetics model, our previous study [34] already verified whether at a low or high ER value, the numerical and experimental wall-pressure distributions fitted well. Meanwhile, Li et al. [31,35] successfully simulated dual-mode flame propagation processes by a 3-D URANS method with this model.

In this paper, supersonic turbulent reactive flows were modeled by solving the 3-D unsteady single-phase multi-species RANS equations, including the continuity and momentum equations, and additional scalar transport equations of turbulent quantities, energy and species. A finite volume method was applied to discretize the equations. Density and convection terms were discretized by the second-order upwind scheme. Pressure and diffusion terms were discretized by the second-order central differencing scheme. Temporal discretization was performed by the second-order backward Euler implicit scheme. The cell center gradients were computed by the least-squares cell-based method. The minmod slopelimiter was utilized for the total-variation-diminishing (TVD) properties. Turbulence closure was achieved by Menter's shear-stress transport (SST) k- $\omega$ model [36], including viscous heating, compressible effect and low-Reynolds-number corrections. The Prandtl number was 0.85 , and the turbulent Schmidt number was 0.7. The ethylene-air combustion mixture was taken as an ideal gas, and thermophysical mixture properties used the mass-weighted mixing law. For each species, the specific heat was defined as a piecewise polynomial function of temperature from NASA database that is valid up to $5000 \mathrm{~K}$, and the thermal conductivity and viscosity were calculated by kinetic theory. The two-step kinetics model above was adopted for ethylene-air reactions. Turbulence/chemistry interactions were treated by the finite-rate/eddy-dissipation model.

\subsection{Computational grids and boundary condition types}

A multi-block hexahedron grid scheme was adopted for numerical accuracies. Computations were performed on a quarter of the combustor considering symmetries. Three grids, containing 1.91 million (coarse), 3.89 million (medium), and 5.86 million (fine) cells, respectively, were tested to verify grid convergence. Wall-normal distance from each first-layer cell center was set to $0.001 \mathrm{~mm}$ for $y^{+}<1$. All grids had more than 20 layers in the domain of $y^{+}<10$, ensuring accurate simulations of turbulent boundary-layers. Fig. 2 presents the medium grid.

For both the isolator and injector inlets, the turbulent intensity and turbulent viscosity ratio were specified as $1 \%$ and 1.0 , respectively, and the inlet boundary-layers were ignored. The isolator inflow was supersonic. Thus, the static pressure, stagnation pressure and stagnation temperature were all specified. The fuel was injected into the combustor as choked sonic flows. Thus, only

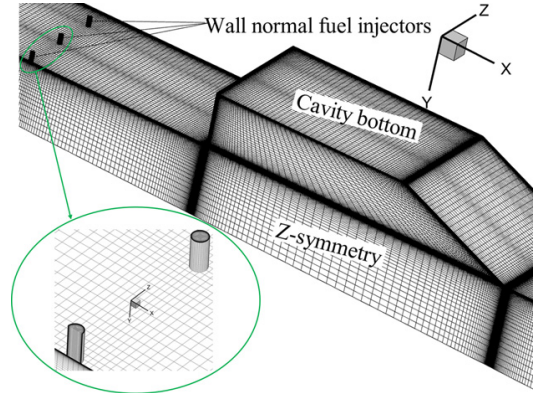

Fig. 2. Medium grid near the cavity.

the stagnation pressure and stagnation temperature were specified at the injector inlet. Supersonic extrapolation was adopted at the combustor outlet. Conservative interpolation was conducted at the combustor/injector interface. Mirror reflection was implemented on each symmetry. All walls were stationary, no-slip and adiabatic.

\subsection{Computational procedure}

The time-step sizes and sub-iteration number per time-step were carefully specified to achieve a compromise between computational costs and numerical accuracies. The time-step sizes were carefully chosen for independences, between $1 \times 10^{-6} \mathrm{~s}$ and $1 \times$ $10^{-5} \mathrm{~s}$, and mostly $4 \times 10^{-6} \mathrm{~s}$. The maximum sub-iteration number was set as 50 for all URANS computations. Two ER regulation methods were commonly used, i.e., linearly $[2,7,9]$ or abruptly [6, 10,37]. The former method achieved combustion-state evolutions by continuous ER variations, but the effect of different variation rates was not strictly clear. To observe stabilized flame and unsteady combustion oscillations at each ER value and combustionstate evolutions when the ER varied, the current study regulated the ER abruptly in a piecewise constant manner. A similar manner was adopted in the study of shock wave reflection hysteresis [38]. The first URANS solution was at the combustion flow $E R=0.10$. Its initial flow field was the nonreactive RANS solution at $\mathrm{ER}=0.10$. To initialize ignition, high-temperature mixtures were patched in the cavity. For any other solution, the ER was varied abruptly to its next value, and kept constant for thousands of time steps until the thrust was stable or periodically oscillating, which indicated a stabilized-flame solution at this ER.

\subsection{Grid convergence and time-step independence verifications}

Grid convergence was verified by URANS computations at ER = 0.78 , using the three grids above and the same time-step size of $2 \times 10^{-6} \mathrm{~s}$, which was small enough. From the point of engine designs, the thrust is a representative performance parameter. Fig. 3 shows the periodic thrust oscillations. The coarse-grid solution differed a lot from the other two, while the medium-grid solution was relatively more similar to the fine-grid solution. Thus, the medium grid ensured enough numerical accuracies, and all the other solutions were based on this grid.

Time-step independences were also verified for all solutions. Fig. 4 shows the periodic thrust-time curves at $E R=0.78$ using the time-step sizes of $1 \times 10^{-6} \mathrm{~s}, 2 \times 10^{-6} \mathrm{~s}$ and $4 \times 10^{-6} \mathrm{~s}$ respectively. The three curves have similar shapes, but the curves of $2 \times$ $10^{-6} \mathrm{~s}$ and $1 \times 10^{-6} \mathrm{~s}$ were closer in phase, indicating the same frequency of about $830 \mathrm{~Hz}$. Because low-frequency $(\leq 1000 \mathrm{~Hz}$ ) large-amplitude oscillations were concerned, $2 \times 10^{-6} \mathrm{~s}$ was small enough for numerical accuracy. 


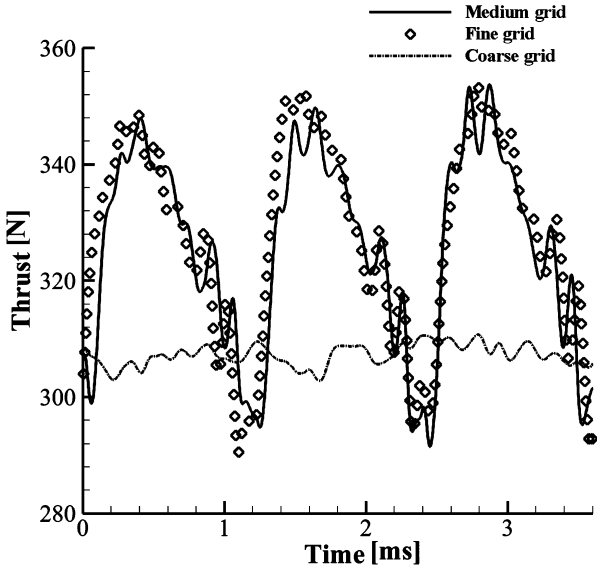

Fig. 3. Thrust-time curves for $E R=0.78$ using different grids. Time step size: $2 \times$ $10^{-6} \mathrm{~s}$.

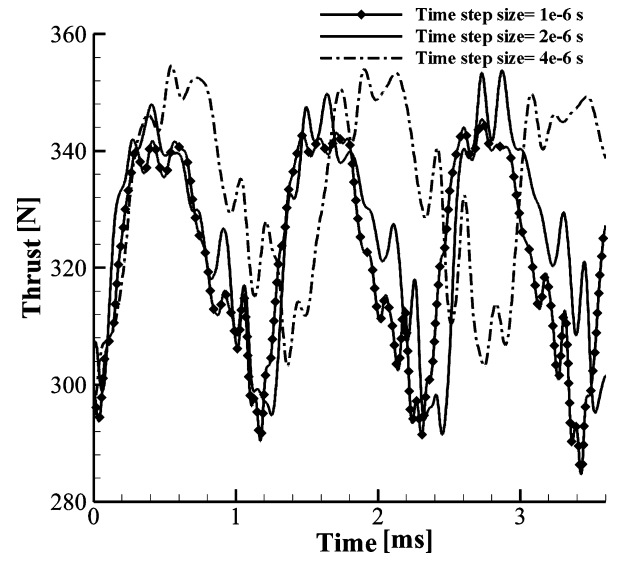

Fig. 4. Thrust-time curves at $E R=0.78$ using different time-step sizes, respectively.

\section{Results and discussions}

\subsection{Combustion hysteresis loops}

For the reciprocating ER variation process under the constant isolator inflow condition, Fig. 5 presents the time-averaged wall pressure at point $\mathrm{K}$ as a function of ER. This chart illustrates two combustion hysteresis loops, while former studies [5-9] observed only one. The first loop was in the ER range between 0.39 and 0.78 , and the second was between 0.80 and 0.92 . The variation percentage between two values is defined as the difference value divided by the larger value. For instance, at $E R=0.49$, the time-averaged wall pressure varied as much as about $67 \%$ based on whether the ER was historically increasing or decreasing. But at $E R=0.83$, it varied only about $19 \%$. Thus, the wall-pressure catastrophe degree of the first loop was much larger than the second.

Fig. 6 displays the thrust calculated by integrating both the combustor wall pressures and friction stresses. At $E R=0.49$, the time-averaged thrust varied as much as about $69 \%$ based on whether the ER was historically increasing or decreasing. But at $\mathrm{ER}=0.83$, it varied only about $7 \%$. In comparison, the time-averaged values of the thrust and the wall pressure at point $\mathrm{K}$ had similar trends, but differed a little especially in the second loop. Meanwhile, Figs. 5 and 6 illustrate oscillation amplitudes of the thrust and wall pressure also differed a little.

Ramjet/scramjet mode is commonly discerned by the minimum Mach number of the one-dimensional combustor flow $\left(M a_{\min }\right)$ [1]. If $M a_{\min }<1.0$, the combustor operates in ramjet mode, otherwise in scramjet mode. Scramjet mode can be subdivided into scramjet

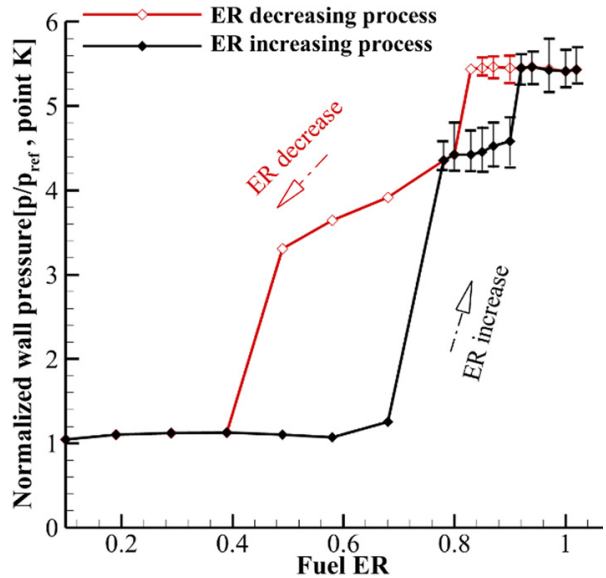

Fig. 5. Pressure vs. ER. Hollow/solid diamond symbols: time-averaged values. Short horizontal lines: upper and lower oscillation limits, and oscillations of the unmarked cases are ignorable.

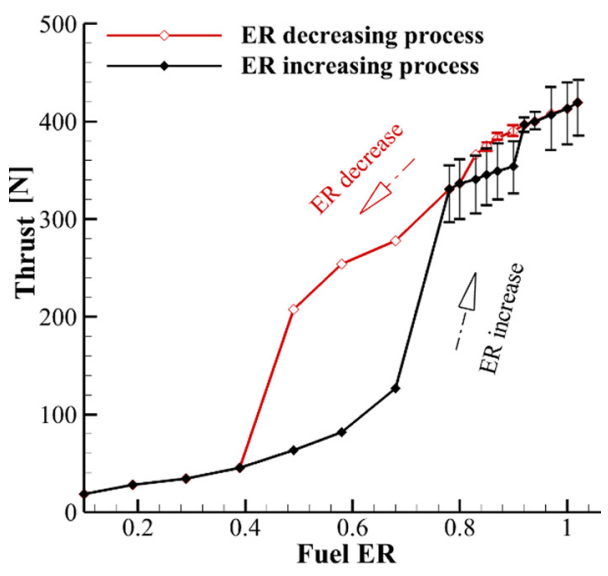

Fig. 6. Thrust vs. ER.

mode with a pre-combustion shock train (separated scramjet mode [39]) and shock-free scramjet mode. In a practical engine, "precombustion" signifies the region upstream the fuel injectors, and "shock-free" signifies no pre-combustion shock train. The mass-flux weighted averaging method is commonly used to achieve $M a_{\min }$ from numerical data [10,29]. By this method, combustion states at all ERs were recognized in scramjet modes. Furthermore, the existence of a pre-combustion shock train can be suggested by an obvious decrease of $M a_{i e}$ as shown in Fig. 7, compared to the inflow Mach number 3.1. Consequently, this chart indicates the first hysteresis loop occurred between the separated and shockfree scramjet modes, and the second was between two different patterns of separated scramjet modes. These two hysteresis behaviors differed from former studies [5-8], which were hysteresis of mode transitions including ramjet modes by one-dimensional estimates of thermal choking.

Fig. 8 presents the thrust-to-THRR ratio, which represents the thermal conversion efficiency from chemical energy to propulsion. According to Brayton Cycle analyses [40,41], a higher combustor wall pressure indicates a higher thermal efficiency, which also means a higher thrust as shown in Fig. 6. Based on Figs. 5-8, the separated and shock-free scramjet modes are equivalent to the intensive-combustion (higher thrust) and weak-combustion modes, respectively. Besides, Figs. 5-8 implies a combustion state in the second loop was in weak-oscillation mode or intensive-oscillation mode depending on historical ER variation directions. While different parameters display different oscillation limits, the concerned thrust performance was certainly influenced by historical ER vari- 


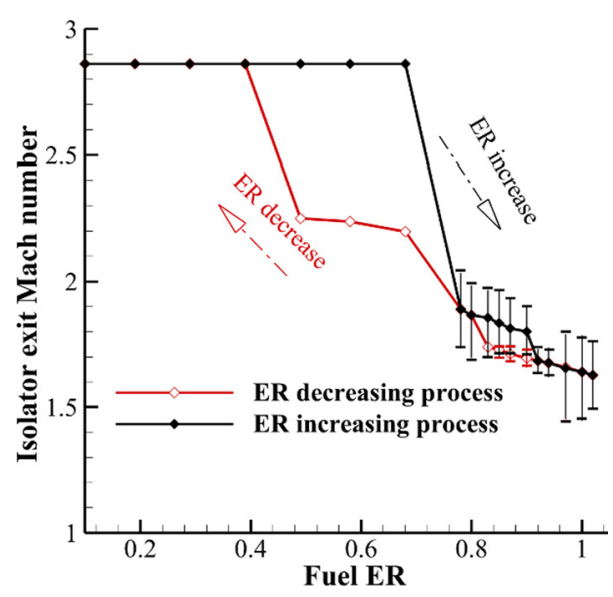

Fig. 7. $M a_{i e}$ vs. ER.

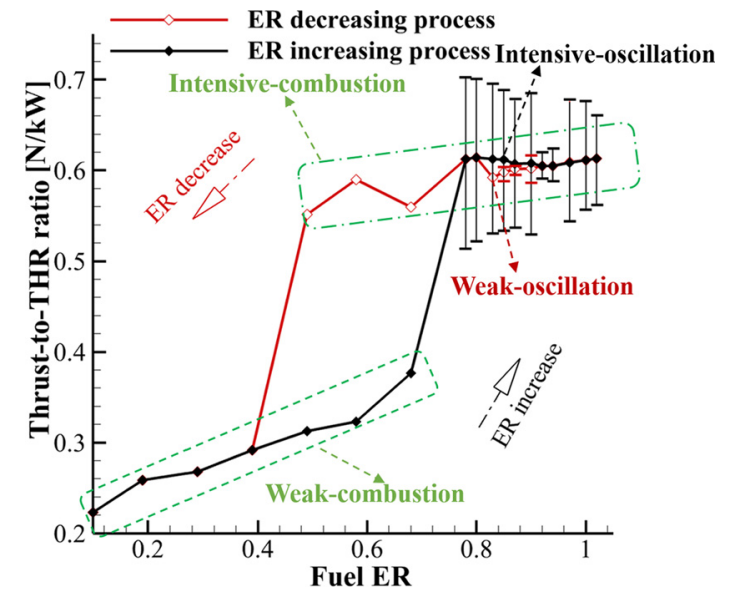

Fig. 8. Thrust-to-THRR ratio vs. ER.

ation directions. The FFT analyses of different oscillation states suggested dominant frequencies in the range expected for thermoacoustic or periodic fluid dynamic instabilities $(100 \sim 1000 \mathrm{~Hz})$. The low-frequency oscillation mechanism is further explained in Section 3.3.

\subsection{Transition of different flame stabilization modes: mechanism of the first hysteresis}

Dual-mode transition behaviors of the first hysteresis loop have been illustrated in Section 3.1. When the ER increased from 0.68 to 0.78 , combustion mode transited from shock-free scramjet mode to separated scramjet mode, along with the transition from weakcombustion mode to intensive-combustion mode. The inverse transition occurred when the ER decreased from 0.49 to 0.39 . The different critical ERs of the two mutually inverse transitions resulted in the first hysteresis. This section mainly illustrates the ER-increasing transition process from the viewpoint of combustion flow structures. The ER-decreasing transition is introduced briefly in this section ending.

Fig. 9 presents the evolutions of THRR and $M a_{i e}$ during the ERincreasing transition. Before the intensive-combustion mode was achieved at $\mathrm{ER}=0.78$, the combustion underwent two stages. In the first stage $(t 1 \sim t 2)$, because of ER increase, THRR fluctuated erratically and tended to increase. To some extent, the catastrophe occurred, showing the abrupt increase of THRR and the abrupt decrease of $M a_{i e}$, which implied the abrupt occurrence of a precombustion shock train. In the second stage $(\mathrm{t} 2 \sim \mathrm{t} 3), M a_{i e}$ fluctuated erratically, and shown an intensive interaction with the THRR,

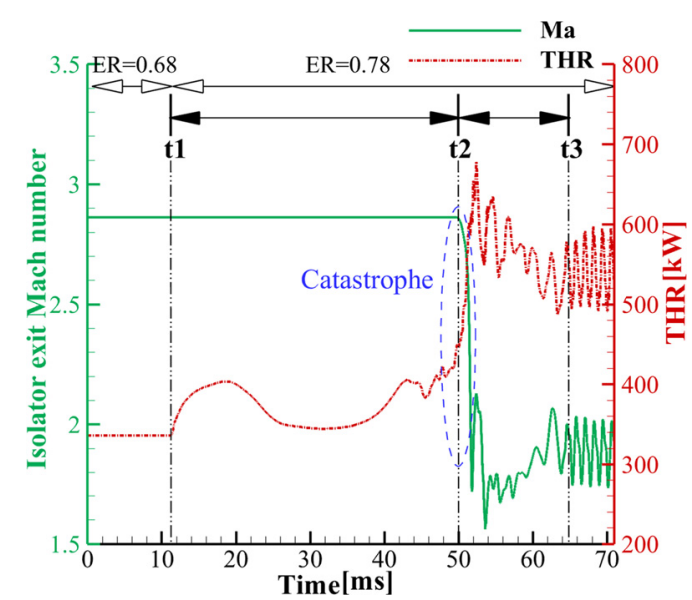

Fig. 9. Time histories of THRR and $M a_{i e}$ (ER from 0.68 to 0.78).

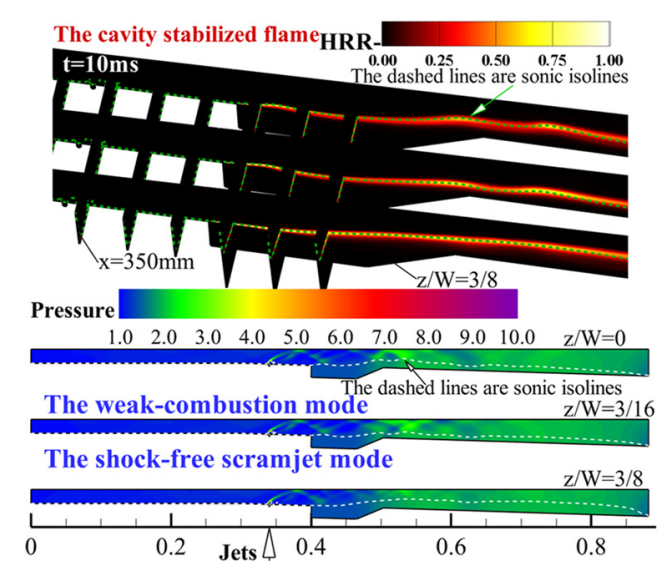

Fig. 10. Typical HRR and pressure distributions at $E R=0.68$ (ER-increasing). The time refers to Fig. 9. HRR is normalized by $12000 \mathrm{MW} / \mathrm{m}^{3}$. Pressure is normalized by isolator inflow pressure. (For interpretation of the colors in the figure(s), the reader is referred to the web version of this article.)

which implied the interaction of the pre-combustion shock train and the intensive combustion heat release. This chart indicates the occurrence of a pre-combustion shock train had a great importance to achieve an intensive-combustion mode.

Fig. 10 presents typical HRR and pressure distributions at $\mathrm{ER}=0.68$. Sonic isolines are depicted in this figure, which implies subsonic regions. Separations must occur in subsonic regions. Consequently, the ignorable separation upstream the fuel injectors illustrates a typical shock-free scramjet mode. The flame was in the cavity shear-layer stabilized mode, in which the flame was anchored in the cavity shear-layer region, and spread as a continuous structure into the main flow at an angle matching the premixed flame theory. This chart also shows a weak-combustion mode, in which most heat release occurred in the downstream low-speed near-wall regions, and the pressure rise upstream the cavity was negligible.

According to one-dimensional flow equations, combustion heat release raises density in a supersonic flow, but reduces density in a subsonic flow. Thus, combustion results in enlarged subsonic lowspeed regions. Meanwhile, combustion heat release raises pressure in a supersonic flow. A shock train will occur if combustion generates sufficiently high pressure. The shock train in return will generate flow separations, and reduce flow speed, and promote combustion. In other words, combustion creates an effective thermal blockage to the flow, which in return benefits the flame propagations. 

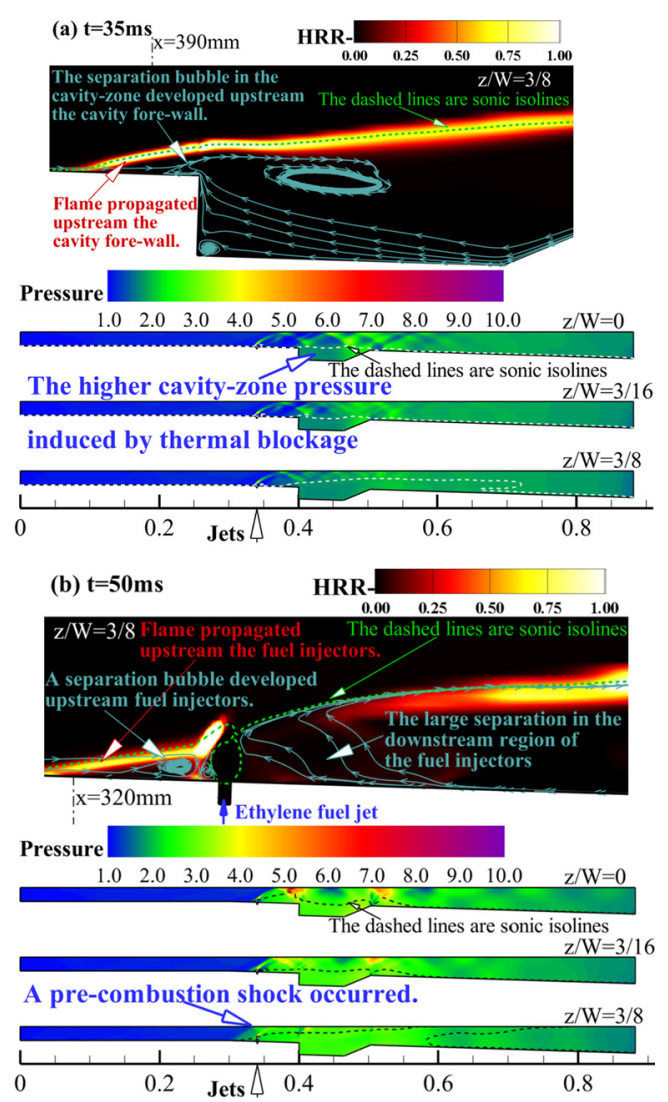

Fig. 11. Typical HRR and pressure evolutions when ER increased from 0.68 to 0.78 . The instructions refer to Fig. 10.

Fig. 11 shows typical HRR and pressure evolutions when the ER increased from 0.68 to 0.78 . At first, combustion heat release increased because of ER increase. Thus, the cavity-zone pressure was obviously raised. Due to the sidewall boundary and corner separation effects, the higher backpressure influenced upstream, and the separation bubble in the cavity-zone developed, and generated separation upstream the cavity fore-wall. As shown in Fig. 11a, the flame propagated upstream in the low-speed region beside the separation bubble, which triggered the mode transition. Compared to Fig. 10, the shock train became slightly visible, while the flow upstream the fuel injectors still remained shock-free. Then, the combustion further intensified because of positive interactions between the flow separation and flame propagation. As shown in Fig. 11b, the flow separation in the downstream region of the fuel injectors was quite large, and the flame propagated further upstream. Even upstream the fuel injectors, a separation bubble occurred, and some flame propagated in the adjacent low-speed region, and a pre-combustion shock also occurred (catastrophe).

Fig. 12 presents typical HRR and pressure distributions at $\mathrm{ER}=0.78$. The obvious pre-combustion shock train and the large flow separation in the upstream region of the fuel injectors illustrate a typical separated scramjet mode. The flame was in the jet-wake stabilized mode, in which the flame was anchored in the jet-wake upstream the cavity, and the flame front had a curved shape. This chart also shows an intensive-combustion mode, in which much heat release occurred in the jet-wake upstream the cavity fore-wall, and a significant pressure rise was detected in the upstream region of the fuel injectors.

The combustion flow structure evolutions of the mode transition when the ER increased from 0.68 to 0.78 is illustrated above, showing as the flame stabilization mode transition from the cavity shear-layer stabilized to the jet-wake stabilized. Owing to the existence of the large flow separation in the upstream region of

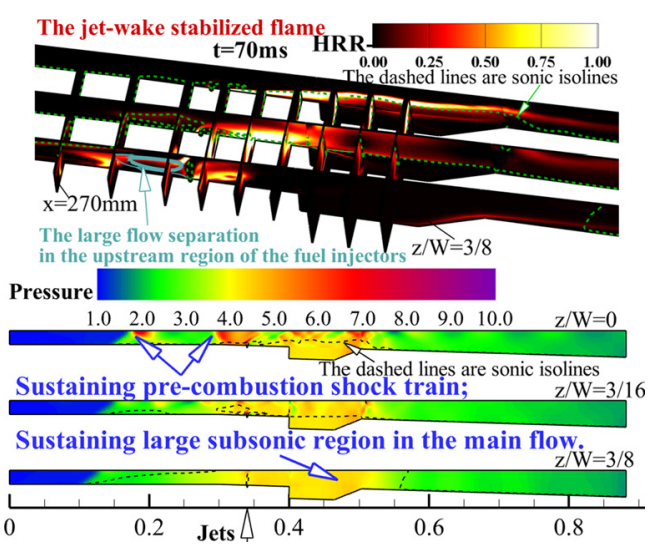

Fig. 12. Typical HRR and pressure distributions at $E R=0.78$. The instructions refer to Fig. 10.

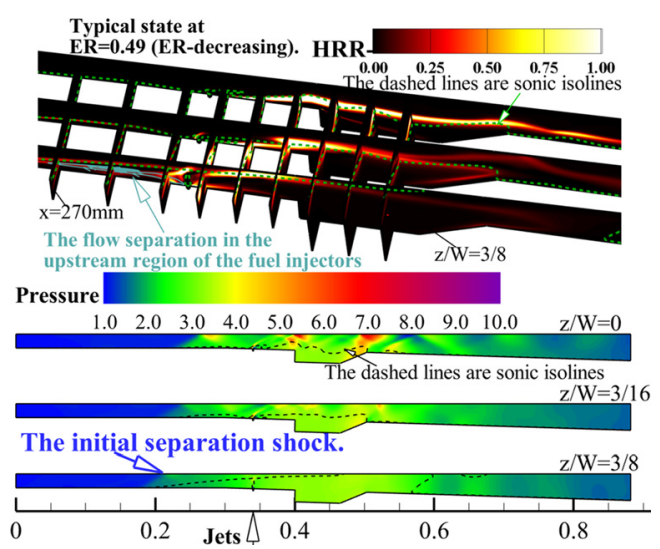

Fig. 13. Typical HRR and pressure distributions at $E R=0.49$ (ER-decreasing). The instructions except the time refer to Fig. 10.

the fuel injectors, the flow speed was rather low, which promoted combustion. When the ER decreased from 0.78 to a lower value, the flame would remain in the jet-wake stabilized mode until the ER was rather low. The inverse transition occurred when the ER decreased from 0.49 to 0.39 , and it is illustrated briefly in Fig. 13 .

Fig. 13 presents typical HRR and pressure distributions at $\mathrm{ER}=0.49$ (ER-decreasing). The pre-combustion shock train actually had only one shock reflection. According to the free interaction theory of shock/boundary-layer [42], the initial shock intensity was determined by its upstream inflow. As the isolator inflow condition was constant, the initial shock kept its intensity almost constantly if it existed. But if the combustion heat release didn't generate sufficiently high pressure, the initial shock and its separation bubble ahead of the injectors would disappear, and the flow speed would be supersonic, and the jet-wake flame would be blown downstream. This was the reason why the flame transited to the cavity shear-layer stabilized mode when the ER decreased to 0.39 .

\subsection{Transition of different combustion oscillation modes: mechanism of the second hysteresis}

Sections 3.1 suggests the second hysteresis occurred as dualmode transition behaviors between two different patterns of separated scramjet modes, which weren't easily understandable. While the flame was always in the jet-wake stabilized mode as confirmed in Section 3.2, unsteady oscillation states depended on historical ER variation directions. A similar phenomenon had been experimentally observed in a high-temperature gas-portfire-based combustor [6,43]. As shown in Fig. 14, the flame was always stabilized 


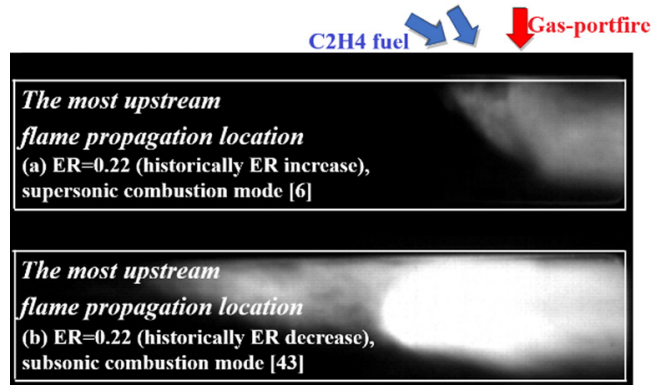

Fig. 14. Critical combustion luminosity images in a gas-portfire-based combustor [6, 43].

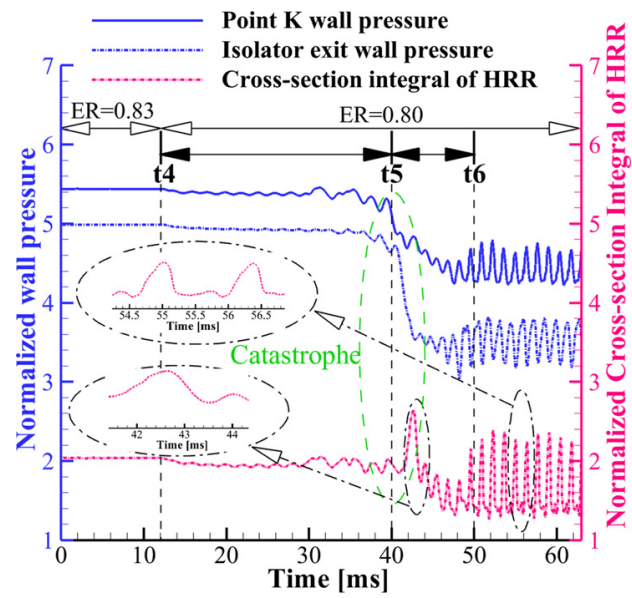

Fig. 15. Time histories of wall pressures at $x=300 \mathrm{~mm}$ and $450 \mathrm{~mm}$, respectively, and the CIHRR at $\mathrm{x}=450 \mathrm{~mm}$ (ER from 0.83 to 0.80 ). Pressures are normalized by isolator inlet pressure. The CIHRR is normalized by $1 \mathrm{MW} / \mathrm{m}$.

in the gas-portfire location, but different critical flame distributions indicate different oscillation states at the same ER.

When the ER increased from 0.90 to 0.92 , the combustion transited from intensive-oscillation mode to weak-oscillation mode. The inverse transition occurred when the ER decreased from 0.83 to 0.80 . The different catastrophe ERs of the two mutually inverse transitions resulted in the second hysteresis. To obtain a better understanding of oscillation mode transitions, combustion flow structure evolutions are illustrated in this section, and the lowfrequency oscillation mechanism is elucidated. Because of a more obviously abrupt change of oscillation state as shown in Figs. 5-8, the ER-decreasing transition is illustrated first in detail. The ERincreasing transition is briefly introduced in this section ending.

Fig. 15 presents the evolutions of wall pressures at $\mathrm{x}=300 \mathrm{~mm}$ and $450 \mathrm{~mm}$, respectively, and the cross-section integral of HRR (CIHRR) at $\mathrm{x}=450 \mathrm{~mm}$ during the ER-decreasing transition. Before the intensive-oscillation mode was achieved at $E R=0.80$, the combustion underwent two stages. In the first stage $(t 4 \sim t 5)$, due to ER decrease, the pressure and CIHRR slightly decreased, and the oscillations tended to be slightly obvious. To some extent, the catastrophe occurred as an abruptly increased CIHRR oscillation intensity, and the abrupt decrease of pressures indicated the precombustion shock train structure catastrophe. In the second stage $(t 5 \sim t 6)$, the pressures and CIHRR oscillated erratically in-phase, and oscillations grew stronger and stronger. This chart indicates the pre-combustion shock train structure catastrophe played an important role in the combustion oscillation mode transition process.

The isolator inflow temperature was about $630 \mathrm{~K}$, which couldn't support pure auto-ignition of the ethylene fuel. Thus, the jetwake stabilized flames were partially premixed combustion flames,

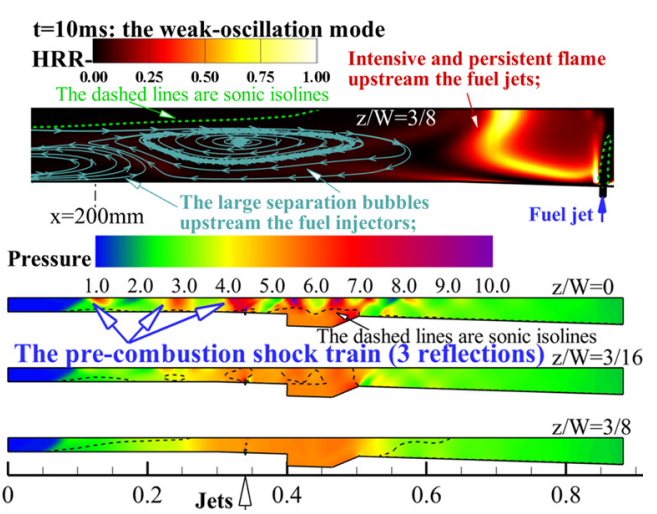

Fig. 16. Typical HRR and pressure distributions at $E R=0.83$ (ER-decreasing). The time refers to Fig. 15. The other instructions refer to Fig. 10.

which consists of initial fuel decompositions, a preheat layer and a heat release zone (premixed flame and downstream diffusion flame) [44]. Combustion produces heat release and enlarges subsonic low-speed regions, which in return can prolong fuel residence time, and provide heat and hot radicals to sustain combustion. The fuel-air mixing/reaction processes in adjacent regions are mutually affected, and diffusions of heat and hot radicals are important for flame stabilizations. Particularly, flame in the low-speed region nearby the separation bubbles ahead of the fuel injectors is relatively more stable compared to flame in other regions because of lower flow speeds, and can promote the whole flame stabilization.

Fig. 16 presents typical HRR and pressure distributions at $\mathrm{ER}=0.83$. The pre-combustion shock train had three reflections with almost no location and intensity variations. The separation bubbles ahead of the fuel injectors were quite large, and flame in the adjacent low-speed region was fairly intensive and stabilized, which provided a fairly stable source of heat and hot radicals for downstream flame stabilization. Consequently, the combustion was in weak-oscillation mode.

Fig. 17 shows typical HRR and pressure evolutions when the ER decreased from 0.83 to 0.80 . At first, combustion receded a little due to ER decrease. Fig. 17a displays that the separation bubbles ahead of the fuel injectors remained almost unchanged, and the flame ahead of the injectors weakened and receded backward slightly, which acted as a less reliable source of heat and hot radicals for downstream stabilized combustion. When the combustion further receded to some extent, the backpressure was not high enough, and the pre-combustion shock train underwent a structure catastrophe. As shown in Fig. 17b, the last pre-combustion shock reflection passed through the injection bow shock. The separation bubbles ahead of the fuel injectors were smaller and obviously closer to the injectors, and the adjacent low-speed region was smaller, and the flame ahead of the injectors weakened and receded backward notably, which could no longer provide a reliable source of heat and hot radicals for downstream flame stabilization. Thus, the combustion couldn't sustain in weak-oscillation mode any more.

Fig. 18 presents typical HRR and pressure distributions at $E R=0.80$. The pre-combustion shock train had two reflections. The shock intensities fluctuated obviously, but reflection locations kept almost stationary. The separation bubble ahead of the fuel injectors was relatively smaller and more unsteady, and the flame ahead of the injectors was weak and relatively more unsteady with reciprocating propagations, which couldn't provide enough heat and hot radicals for steady downstream combustion. Thus, the combustion at $\mathrm{ER}=0.80$ was achieved as intensive-oscillation mode.

Lin et al. $[18,45]$ theoretically explained thermo-acoustic instabilities as feedback loops in the subsonic region bounded by the 

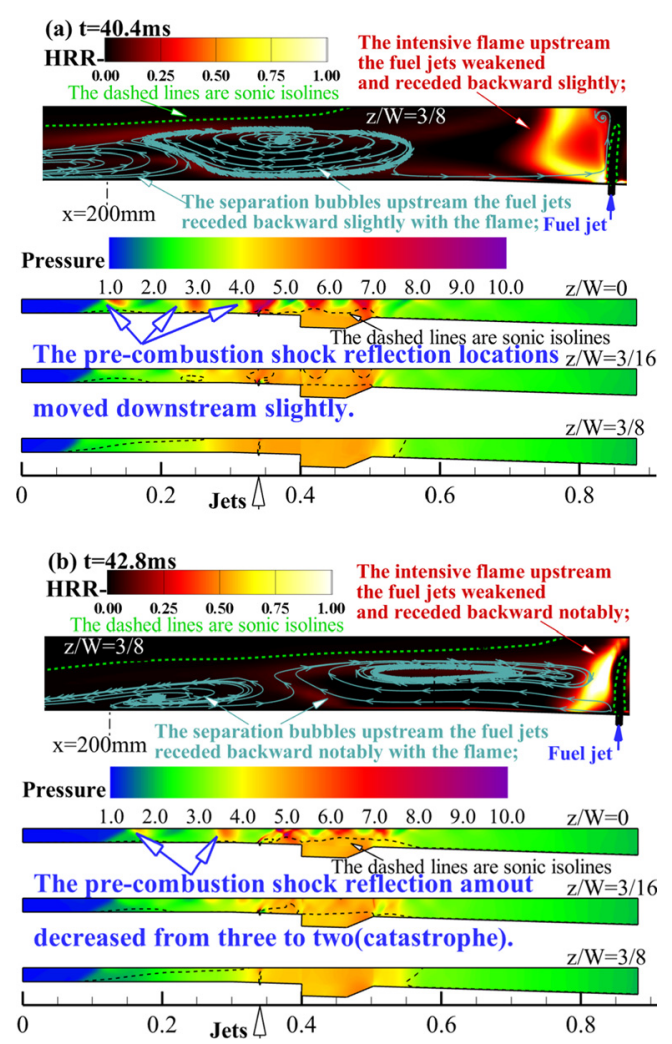

Fig. 17. Typical HRR and pressure evolutions when ER from 0.83 to 0.80 . The instructions refer to Fig. 16.
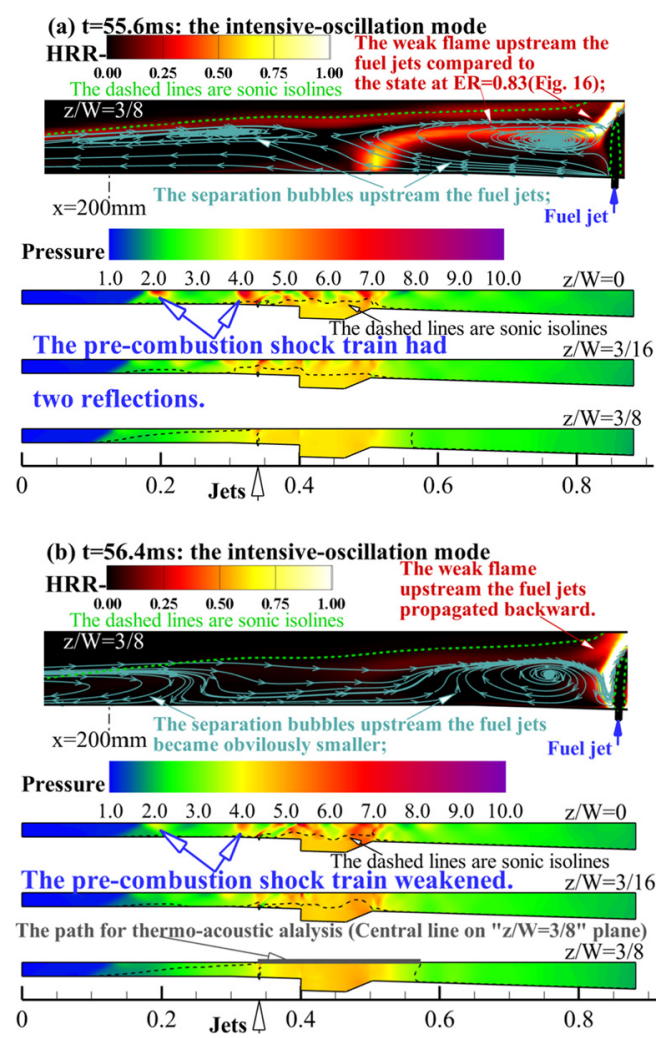

Fig. 18. Typical HRR and pressure distributions at $E R=0.80$. The instructions refer to Fig. 16.

pre-combustion normal shock location and the downstream thermal throat based on the quasi-one-dimensional flow assumption.
Table 1

\begin{tabular}{lcl}
$\begin{array}{l}\text { Estimated } \\
\text { (ER=0.80. Four typical times). }\end{array}$ & frequencies \\
\hline Time ms & $f_{a-a} \mathrm{~Hz}$ & $f_{a-c} \mathrm{~Hz}$ \\
\hline 55.6 & 799 & 649 \\
56.0 & 853 & 612 \\
56.4 & 862 & 640 \\
56.8 & 896 & 691 \\
Arithmetic average & 852 & 648 \\
\hline
\end{tabular}

But this explanation was only applicable in ramjet mode, not in scramjet mode. At $E R=0.80$, the in-phase oscillations of pressure and heat release shown in Fig. 15 was a typical characteristic of thermo-acoustic instability. Interactions of acoustic waves and fuel injection/mixing processes mutually effect combustion processes. And because acoustic waves can only propagate upstream in subsonic regions to form a one-dimensional propagation feedback loop [18], the current study bounded a subsonic region by sonic-points on the geometric central-line shown in Fig. 18b. The thermo-acoustic frequencies can be estimated, respectively, as

$$
\begin{aligned}
\tau_{a-a} & =\int_{x_{s}}^{x_{e}} d x /(a-u)+\int_{x_{s}}^{x_{e}} d x /(a+u) \\
\tau_{a-c} & =\int_{x_{s}}^{x_{e}} d x /(a-u)+\int_{x_{s}}^{x_{e}} d x / u
\end{aligned}
$$

where $x_{s}$ and $x_{e}$ represent the two sonic-point locations, respectively, and $a$ represents local acoustic-velocity, and $u$ is locally streamwise flow-velocity, and subscripts " $a-a$ " and " $a-c$ " denote acoustic-acoustic and acoustic-convective feedback loops, respectively.

By using Equations (1) and (2) at several times over a dominant oscillation period, the estimated thermo-acoustic frequencies at $\mathrm{ER}=0.80$ were achieved as $852 \mathrm{~Hz}$ and $648 \mathrm{~Hz}$, respectively, as shown in Table 1. Meanwhile, the FFT analysis of wall-pressure data revealed a dominant frequency of $739 \mathrm{~Hz}$, which matched the estimated thermo-acoustic frequencies reasonably well. Thus, Lin's explanation of thermo-acoustic combustion instabilities can be generalized to the separated scramjet mode.

The combustion flow structure evolutions of the mode transition when the ER increased from 0.83 to 0.80 is illustrated above, and the low-frequency oscillation mechanism is elucidated for a better understanding of oscillation mode transitions. Owing to the small separation bubbles ahead of the injectors at $E R=0.80$, the flame in the low-speed region ahead of the injectors was weak and unsteady, which gave rise to downstream intensive-oscillation combustion. When the ER increased from 0.80 to a higher value, the combustion remained in intensive-oscillation mode until the ER was so high that the pre-combustion shock reflection amount increased by one. This transition occurred when the ER increased from 0.90 to 0.92, and it is illustrated briefly in Fig. 19.

Fig. 19 presents typical HRR and pressure distributions at $\mathrm{ER}=0.90$ (ER-increasing) and $\mathrm{ER}=0.92$, which are similar to the distributions at $\mathrm{ER}=0.80$ and $\mathrm{ER}=0.83$ (ER-decreasing), respectively. At $E R=0.90$, the relatively more weak and unsteady flame ahead of the injectors resulted in intensive-oscillation mode. When the ER increased to 0.92 , the pre-combustion shock train also experienced a structure catastrophe, showing as the increase of shock reflections from 2 to 3 (catastrophe). Consequently, the flow separation ahead of the fuel injectors was obviously larger, and the flame in the low-speed region beside the separation bubbles was fairly steady and intensive, which guaranteed that the combustion at $\mathrm{ER}=0.92$ was in weak-oscillation mode. 


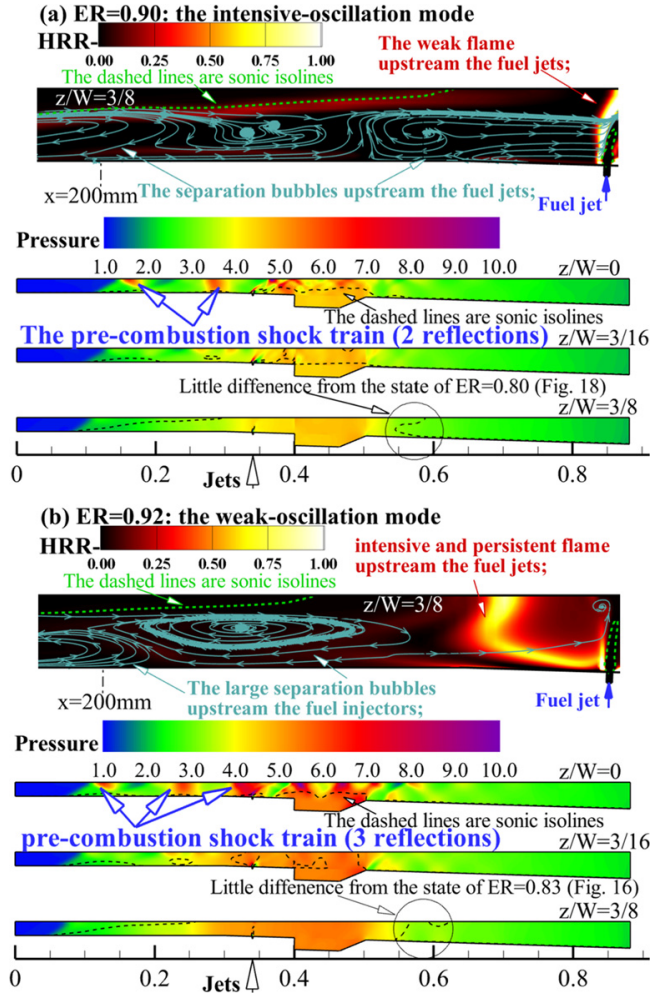

Fig. 19. Typical HRR and pressure distributions at (a) $E R=0.90$ (ER-increasing) and (b) $E R=0.92$, respectively. The instructions except the time refer to Fig. 10 .

\section{Conclusion}

The effect of ethylene fuel ER variation directions on combustion states in a dual-mode scramjet combustor was numerically investigated. The combustor employed transverse wall fuel injectors and downstream cavity flameholders without pilot fuel. Computations were conducted at flight Mach 6.0 enthalpy. The isolator inflow Mach number was 3.1, and static pressure, stagnation pressure and stagnation temperature were $53 \mathrm{kPa}, 2622 \mathrm{kPa}$ and $1656 \mathrm{~K}$, respectively. The ER was regulated abruptly in a piecewise constant manner, from 0.10 to 1.02 , and then back to 0.10. A 3-D URANS method with a recognized two-step kinetics model was adopted to simulate flame propagations. The main conclusions are summarized as follows:

1) For the reciprocating ER variation process under the constant isolator inflow condition, the current study observed two combustion hysteresis loops, while former studies [5-9] observed only one.

2) The first hysteresis occurred between separated and shockfree scramjet modes based on steady quasi-one-dimensional combustor flow assumptions, and the second was between two different patterns of separated scramjet modes. These two hysteresis behaviors differed from former studies [5-8], which were hysteresis of mode transitions including ramjet modes by one-dimensional estimates of thermal choking.

3 ) The first hysteresis was attributed to the flame stabilization mode transitions between the cavity shear-layer stabilized mode and the jet-wake stabilized mode, along with the transition hysteresis of a pre-combustion shock train's establishment and vanishment. The flame stabilization locations were greatly influenced by the flow separation states ahead of the fuel injectors, and the flow separations were in return determined by the flame distributions.

4) The second hysteresis was attributed to the combustion oscillation mode transitions between weak-oscillation mode and intensive-oscillation mode, along with the transition hysteresis of shock reflection amount increase and decrease of the precombustion shock train structure. Flame in the low-speed region beside the separation bubbles ahead of the fuel injectors provided heat and hot radicals for downstream flame stabilization, and the pre-injector flame intensity greatly influenced the combustion oscillation states.

5) A low-frequency oscillation (100 1000 Hz) mechanism in the separated scramjet mode was elucidated based on Lin's explanation of thermo-acoustic combustion instabilities, which was once only applicable in ramjet modes [18].

The first hysteresis influenced the thrust performance more greatly than the second hysteresis. According to our knowledge, this hysteresis might be avoided by optimizations of the combustor configuration including the divergent duct angle, the location of the cavity and the fuel injectors, etc, or by utilizing additional methods such as a high-temperature gas-portfire [6] or air throttling [46]. The second hysteresis was relatively small but still not acceptable, and further study remains to be done to eliminate this hysteresis. These conclusions provide updated insight into mode transitions and combustion hysteresis. They're expected to be helpful for system design of general dual-mode scramjet combustors with transverse wall fuel injectors and downstream cavity flameholders.

\section{Declaration of competing interest}

No conflict of interest.

\section{Acknowledgements}

This work was supported by the National Natural Science Foundation of China (Grant Nos. 11672309 and 11472279).

\section{References}

[1] W.H. Heiser, D.T. Pratt, Hypersonic Airbreathing Propulsion, AIAA Education Series, AIAA, 1994.

[2] B.G. Xiao, Y. Tian, et al., Experimental and numerical investigations of combustion mode transition in a direct-connect scramjet combustor, Aerosp. Sci. Technol. 46 (2015) 331-338.

[3] C.L. Zhang, J.T. Chang, et al., Pressure rising slope variation accompanying with combustion mode transition in a dual-mode combustor, Aerosp. Sci. Technol. 68 (2017) 370-379

[4] C.L. Zhang, J.T. Chang, et al., Effect of Mach number and equivalence ratio on the pressure rising variation during combustion mode transition in a dualmode combustor, Aerosp. Sci. Technol. 72 (2018) 516-524.

[5] R.D. Rockwell Jr., et al., Experimental study of test-medium vitiation effects on dual-mode scramjet performance, J. Propuls. Power 27 (5) (2011) 1135-1142.

[6] B.X. Wei, X. Xu, et al., Experimental study on combustion mode transition in an aero-ramp based scramjet, AIAA 2012-3923.

[7] W. Bao, J.T. Chang, et al., Dynamic characteristics of combustion mode transitions in a strut-based scramjet combustor model, J. Propuls. Power 29 (5) (2013) 1244-1248.

[8] S.H. Zhu, X. Xu, Experimental study on mode transition of the dual-mode scramjet with two-staged-strut injectors, Proc. Inst. Mech. Eng., G J. Aerosp. Eng. 232 (10) (2018) 1864-1874.

[9] S.H. Zhu, X. Xu, P.F. Ji, Flame stabilization and propagation in dual-mode scramjet with staged-strut injectors, AIAA J. 55 (1) (2017) 171-179.

[10] Y. Zhang, X. Xu, et al., Hysteresis of mode transition in a dual-struts based scramjet, Acta Astronaut. 128 (2016) 147-159.

[11] V.I. Arnol'd, Bifurcation Theory and Catastrophe Theory, Springer, 1999

[12] D.R. Yu, T. Cui, W. Bao, Catastrophe, hysteresis and bifurcation of mode transition in scramjet engines and its model, Sci. China Ser. E 52 (6) (2009) 1543-1550.

[13] D.J. Micka, J.F. Driscoll, Combustion characteristics of a dual-mode scramjet combustor with cavity flameholder, Proc. Combust. Inst. 32 (2009) 2397-2404.

[14] H.B. Wang, M.B. Sun, et al., Combustion modes of hydrogen jet combustion in a cavity-based supersonic combustor, Int. J. Hydrog. Energy 38 (2013) 12078-12089.

[15] T. Mitani, et al., Comparison of scramjet engine performance in Mach 6 vitiated and storage-heated air, J. Propuls. Power 13 (5) (1997) 635-642. 
[16] T. Kouchi, et al., Mechanism and control of combustion-mode transition in a scramjet engine, J. Propuls. Power 28 (1) (2012) 106-112.

[17] S. Nakaya, et al., Ignition and supersonic combustion behavior of liquid ethanol in a scramjet model combustor with cavity flame holder, Proc. Combust. Inst. 35 (2015) 2091-2099.

[18] K.C. Lin, et al., Acoustic characterization of an ethylene-fueled scramjet combustor with a cavity flameholder, J. Propuls. Power 26 (6) (2010) 1161-1169.

[19] Z. Cai, M.B. Sun, et al., Effect of cavity geometry on fuel transport and mixing processes in a scramjet combustor, Aerosp. Sci. Technol. 80 (2018) 309-314.

[20] Z. Cai, M.B. Sun, et al., Effect of cavity fueling schemes on the laser-induced plasma ignition process in a scramjet combustor, Aerosp. Sci. Technol. 78 (2018) 197-204.

[21] J. Noh, et al., Numerical simulation of auto-ignition of ethylene in a scramjet combustor with air throttling, AIAA 2010-7036.

[22] L. Tian, et al., Quasi-one-dimensional multimodes analysis for dual-mode scramjet, J. Propuls. Power 30 (6) (2014) 1559-1567.

[23] A. Saghafian, et al., Large eddy simulations of the HIFiRE scramjet using a compressible flamelet/progress variable approach, Proc. Combust. Inst. 35 (2015) 2163-2172.

[24] G.Y. Zhao, M.B. Sun, et al., Investigation of flame flashback phenomenon in a supersonic crossflow with ethylene injection upstream of cavity flameholder, Aerosp. Sci. Technol. 87 (2019) 190-206.

[25] H.B. Wang, M.B. Sun, et al., Large-Eddy Reynolds-averaged Navier-Stokes simulation of combustion oscillations in a cavity-based supersonic combustor, Int. J. Hydrog. Energy 38 (2013) 5918-5927.

[26] S.J. Lawson, G.N. Barakos, Review of numerical simulations for high-speed, turbulent cavity flows, Prog. Aerosp. Sci. 47 (2011) 186-216.

[27] Y.Y. Han, Y. Tian, et al., Assessment of a hybrid RANS/LES simulation method and URANS method in depicting the unsteady motions of flow structures in a scramjet combustor, Aerosp. Sci. Technol. 72 (2018) 114-122.

[28] J. Messineo, Vortex shedding influence on hybrid rocket pressure oscillations and combustion efficiency, J. Propuls. Power 32 (6) (2016) 1386-1394.

[29] Y. Tian, et al., Numerical study on effect of air throttling on combustion mode formation and transition in a dual-mode scramjet combustor, Aerosp. Sci. Technol. 52 (2016) 173-180.

[30] R.J. Yentsch, D.V. Gaitonde, Unsteady three-dimensional mode transition phenomena in a scramjet flowpath, J. Propuls. Power 31 (1) (2015) 104-122.

[31] J. Li, et al., Ignition transients in a scramjet engine with air throttling part II: reacting flow, J. Propuls. Power 31 (1) (2015) 79-88.
[32] T. Kouchi, et al., Numerical simulations in scramjet combustion with boundarylayer bleeding, J. Propuls. Power 21 (4) (2005) 642-649.

[33] C.K. Westbrook, F.L. Dryer, Simplified reaction mechanisms for the oxidation of hydrocarbon fuel in flames, Combust. Sci. Technol. 27 (1981) 31-43.

[34] X. Wang, F.Q. Zhong, H.B. Gu, X.Y. Zhang, Numerical study of combustion and convective heat transfer of a Mach 2.5 supersonic combustor, Appl. Therm. Eng. 89 (2015) 883-896.

[35] J. Li, et al., Ignition transients in a scramjet engine with air throttling part 1 : nonreacting flow, J. Propuls. Power 30 (2) (2014) 438-448.

[36] F.R. Menter, Two-equation eddy-viscosity turbulence models for engineering applications, AIAA J. 32 (8) (1994) 1598-1605.

[37] J.T. Chang, et al., Experimental investigation of hysteresis phenomenon for scramjet engine, AIAA J. 52 (2) (2014) 447-451.

[38] G. Ben-dor, T. Elperin, H. Li, E. Vasiliev, The influence of the downstream pressure on the shock wave reflection phenomenon in steady flows, J. Fluid Mech. 386 (1999) 213-232.

[39] J.C. Turner, et al., Mode change characteristics of a three-dimensional scramjet at Mach 8, J. Propuls. Power 29 (4) (2013) 982-990.

[40] C.H. Builder, On the thermodynamic spectrum of airbreathing propulsion, in: 1st AIAA Annual Meeting, AIAA, 1964, pp. 64-243.

[41] L.R. James, D.P. Marc, Efficiency of an ideal Brayton cycle with a constantvolume interturbine burner, J. Propuls. Power 31 (3) (2015) 970-975.

[42] D.R. Chapman, D.M. Kuehn, H.K. Larson, Investigation of Separated Flows in Supersonic and Subsonic Streams with Emphasis on the Effect of Transition, NACA Tech. Note No. 3869, 1957.

[43] B.X. Wei, Study of Flame Holder and Mode Transition Technology in a DualMode Ramjet Combustor, Doctor Degree Dissertation, Beihang University, 2010 (in Chinese).

[44] D.J. Micka, Combustion Stabilization, Structure, and Spreading in a Laboratory Dual-Mode Scramjet Combustor, Dissertations \& Theses, Gradworks, 2010.

[45] F.H. Ma, J. Li, V. Yang, Thermoacoustic flow instability in a scramjet combustor, in: 41st AIAA/ASME/SAE/ASEE Joint Propulsion Conference \& Exhibit, AIAA 2005-3824.

[46] Y. Tian, et al., Experimental study on flame development and stabilization in a kerosene fueled supersonic combustor, Aerosp. Sci. Technol. 84 (2019) 510-519. 